\title{
Colon Cancer in Patients Younger Than Age 50 in Kuantan: Case Series
}

Nurul Syazwani Abd Khalid, Muhammad Afiq Kodiron, Mohd Yusof Sainal, Faisel Elagili, Azmi Md Nor

International Islamic University Malaysia Medical Centre, Kuantan Pahang.

Introduction: Colorectal cancer (CRC) is the second most common cancer in Malaysia. In general, patients aged > 65 years comprise the majority of the population with CRC. However, recent reports indicate its increasing incidence in younger populations. We describe 4 cases of early onset colorectal cancer in Kuantan. Materials and method: This is a descriptive study of a small series of patients. Data between 2018 and 2019 were obtained from medical charts. Results: We diagnosed four patients under the age of 50 with colon cancer ( $75 \%$ male), the median age was 36 (23-47), with no comorbidities. None of the patient had family history of colorectal cancer. The majority of our patients presented with complication of tumour ( 2 perforation, 1 obstruction) required emergency surgeries. Only one patient presented with with rectal bleeding and was found to have tumor of the rectosigmoid junction. Two patients had tumor at descending colon and one at the splenic flexure. From this case series, we found there were one patient for every stage of colorectal cancer. Carcinoembryonic (CEA) levels were elevated at baseline in only two patients. Conclusion: Incidence of colon cancer rises among young patients in Kuantan. Further studies are needed to clarify the clinical and biological characteristics of colon cancer, improve its treatment strategies, and promote better outcomes in young patients. 